\title{
Molecular Buffers Permit Sensitivity Tuning and Inversion of Riboswitch Signals
}

Rugbjerg, Peter; Genee, Hans Jasper; Jensen, Kristian; Sarup-Lytzen, Kira; Sommer, Morten Otto Alexander

Published in:

A C S Synthetic Biology

Link to article, DOI:

10.1021/acssynbio.5b00213

Publication date:

2016

Document Version

Publisher's PDF, also known as Version of record

Link back to DTU Orbit

Citation (APA):

Rugbjerg, P., Genee, H. J., Jensen, K., Sarup-Lytzen, K., \& Sommer, M. O. A. (2016). Molecular Buffers Permit Sensitivity Tuning and Inversion of Riboswitch Signals. A C S Synthetic Biology, 5(7), 632-638.

https://doi.org/10.1021/acssynbio.5b00213

\section{General rights}

Copyright and moral rights for the publications made accessible in the public portal are retained by the authors and/or other copyright owners and it is a condition of accessing publications that users recognise and abide by the legal requirements associated with these rights.

- Users may download and print one copy of any publication from the public portal for the purpose of private study or research.

- You may not further distribute the material or use it for any profit-making activity or commercial gain

- You may freely distribute the URL identifying the publication in the public portal 


\title{
Molecular Buffers Permit Sensitivity Tuning and Inversion of Riboswitch Signals
}

\author{
Peter Rugbjerg, Hans Jasper Genee, Kristian Jensen, Kira Sarup-Lytzen, \\ and Morten Otto Alexander Sommer*
}

Novo Nordisk Foundation Center for Biosustainability, Technical University of Denmark, Kogle Allé 6, DK-2970 Hørsholm, Denmark

\section{Supporting Information}

ABSTRACT: Predictable integration of foreign biological signals and parts remains a key challenge in the systematic engineering of synthetic cellular actuations, and general methods to improve signal transduction and sensitivity are needed. To address this problem we modeled and built a molecular signal buffer network in Saccharomyces cerevisiae inspired by chemical $\mathrm{pH}$ buffer systems. The molecular buffer system context-insulates a riboswitch enabling synthetic control of colony formation and modular signal manipulations. The riboswitch signal is relayed to a transcriptional activation domain of a split transcription factor, while interacting DNA-binding domains mediate the transduction of signal and form an interacting molecular buffer. The molecular buffer system enables modular signal inversion through integration with repressor modules. Further, tuning of input sensitivity was achieved through perturbation of the buffer pair ratio guided by a mathematical model. Such buffered signal tuning networks will be useful for domestication of RNA-based sensors enabling tunable outputs and library-wide selections for drug discovery and metabolic engineering.

KEYWORDS: riboswitch, signal tuning, signal inversion, signal processing, biosensor
S ynthetic circuits rely on predictable signal processing at the interface of biological input sensors and output actuators. However, constraints in matching signal input/output (I/O) currently limit the possible functions that can be designed. ${ }^{1}$ Accordingly, the integration of subtle inputs with general stabilizing and modulating networks is needed to successfully actuate complex biological programs. Such approaches may further help provide context insulation and stability toward signal errors inherent in many human-designed biological systems, including multicomponent synthetic computation networks, gene-therapeutic dosage control and metabolic biosensors constitute systems that otherwise require precise and robust signal transmission. ${ }^{2-9}$

A wide variety of natural input sensor types exist, which are overall represented by protein transcription factors ${ }^{10,11}$ and RNA switches. ${ }^{12,13}$ Despite an apparent abundance of candidate input sensors, only a modest number of such regulators are routinely used to build most synthetic circuits, namely proteinbased input sensors such as LacI, LuxR and GAL4.,14-18 RNA switches hold the potential advantage that their ligandrecognizing part, the aptamer, can be tailored synthetically for virtually any molecule using the SELEX technology. ${ }^{19}$ Sophisticated sensor modules have been constructed on the basis of modular assembly of synthetic ${ }^{20}$ and programmable de novo RNA switches. ${ }^{21}$ Switches are limited in a number of aspects: input sensitivity, modularity and whether regulation is positive or negative, although recent progress in the design and understanding of RNA regulators has improved their scope of application. ${ }^{14,22-27}$

Specific adaptation of the regulatory properties of an RNA switch to fit the needs of a conceived genetic circuit can be made. For instance, change of sensitivity is possible through rational mutagenesis of the switch, ${ }^{25}$ and riboswitch mutants with inverted outputs have been identified through elaborate screenings. ${ }^{28}$ However, these adaptations are challenging and time-consuming. ${ }^{29}$ In contrast, the response curve of transcription factor-based input sensors can be manipulated modularly, e.g., by deploying synthetic signal drains and competitive inhibitors, ${ }^{30,31}$ which render the signal ultrasensitive and can change the input trigger thresholds. Furthermore, modular signal inversion can be achieved based on translational fusions of the sensor to repressor/activator domains. $^{32,33}$

To minimize the need for tailoring riboswitch sensors to their specific conceived actuations, a modular signal-processing system offering control interfaces without need for changing the actual input and output parts is needed. In this study we set out to stabilize and transform ineffective responses of a tetracycline-responsive riboswitch by directing its output signals through a molecular signal buffer network to enable tunable population-wide selection. Such signal processing adds beneficial modular control points for switch-independent

Received: October 27, 2015

Published: May 3, 2016 
changes of input sensitivity and output direction. We therefore adapt the buffer concept to riboswitch regulation through the use of split transcription factors expressed at uneven ratios as buffer pairs.

\section{RESULTS}

Direct control of complex cellular actuations, such as cell survival by riboswitches can sometimes be challenging to achieve due to leaky expression although the same riboswitch with luminescent or fluorescent outputs yields quantitative and repeatable readouts. In spite of these challenges synthetic control of cell survival is required in order to enable library selections for specific phenotypes or to control cell populations in a variety of applications. To assess these challenges we engineered genetic constructs comprising a tetracyclineresponsive riboswitch ${ }^{34}$ that down-regulates translation of the classical yeast selection gene URA3 in Saccharomyces cerevisiae. URA3 mediates 5-fluoroorotic acid (FOA) sensitivity $\left(\mathrm{FOA}^{\mathrm{S}}\right)$, which permits negative selection. Despite a substantial downregulation capacity of the riboswitch at $37-$ fold, ${ }^{34}$ addition of tetracycline input resulted only in a limited improvement of growth with about 5-fold more colonies appearing on $\mathrm{FOA}^{\mathrm{R}}$ spot assay selection plates (Figure 1), similar to average 21 .

\begin{tabular}{lcl}
\hline Genetic circuit & $\begin{array}{c}\text { Colony formation output } \\
\text { Colony formation rate } \\
10^{-5} 10^{-4} 10^{-3} 10^{-2} 10^{-1}\end{array}$ & $\begin{array}{l}\text { Direct riboswitch } \\
\text { response (GFP) }\end{array}$ \\
\hline Direct signal & & \\
\hline R Riboswitch + Riboswitch ligand &
\end{tabular}

Figure 1. Colony formation in response to direct riboswitch-sensed input. Direct relay of the riboswitch signal to URA3 caused poor control of colony formation (strain PRd5), further indicated by Cterminal GFP-tagging of URA3 (strain PRa116). The riboswitch (R) was expressed in the $5^{\prime}$-untranslated region of URA3. Colony formation responses were determined using spot assays with 10-fold serial dilution of equally dense cultures (Methods) and representative examples of triplicate tests shown. GFP output was measured as background-subtracted relative fluorescence units (RFU) measured from a C-terminally GFP-tagged URA3 (PRa116), with error bars depicting standard error $(n=3)$. Plates were SC-leu-trp, with $0.09 \%$ (w/v) FOA in selective medium. $150 \mu \mathrm{M}$ tetracycline was used as ligand in plates, $250 \mu \mathrm{M}$ tetracycline in liquid cultures.

$10^{-4} \pm 4 \cdot 10^{-4}$ colonies by plating, where $150 \mu \mathrm{M}$ tetracycline supplementation increased the number to $103 \cdot 10^{-4}$ colonies \pm $17 \cdot 10^{-4}$ ( \pm standard error, $n=3$ ).

In selections, cells that form colonies in the absence of the input are false positives. Due to single cell variation, these might, e.g., form despite correct population-level URA3 expression level. Equally undesirable are the false-negative cells that fail to form colonies despite receiving input. Both error types limit the possible throughput, e.g., when assaying libraries. We wanted to ensure that URA3 expression was within the dynamic range of cell death and survival. Too high basal URA3 expression would mean that even the downregulated URA3 expression is too high to cause survival due to leakiness (false negatives). Oppositely, too low basal expression would constitutively cause survival (false positives). Since all plated cells did not form colonies with tetracycline (Figure 1), the system appeared to yield false-negative cells. Similarly false- positive cells were indicated due to cells forming colonies in absence of tetracycline (Figure 1).

This simultaneous occurrence of false-positive and falsenegative cells indicated a fundamentally poor signal relay. We subsequently tagged the regulated URA3 C-terminally with GFP, which showed no tetracycline-dependent down-regulation, indicating incompatibility between URA3 and the riboswitch (Figure 1). Such detrimental "part-junction" interference $^{35}$ might, e.g., result from interacting mRNA structures, similar to interaction effects seen between promoters and circuit output, which could be buffered using ligandindependent ribozymes. ${ }^{35}$ Thus, we speculated that an additional regulatory layer might provide important signal insulation, while further functioning as a signal-processing platform to modulate the riboswitch signals.

A Synthetic Molecular Signal Buffer to Effectively Relay the Riboswitch Signal. Biological and chemical systems maintain $\mathrm{pH}$ homeostasis by providing a surplus of interconvertible acid/base species. Their ratio influences $\mathrm{pH}$ and shows the capacity to react with fluctuating molecules to render them dysfunctional, rather than affecting $\mathrm{pH}$, unless a specific threshold equivalence point is reached; e.g., human blood is buffered stably to $\mathrm{pH} \mathrm{7.4} \mathrm{Thus,} \mathrm{we} \mathrm{hypothesized} \mathrm{that} \mathrm{a}$ simple, protein-based signal buffer can be engineered in a similar fashion.

One embodiment of such synthetic signal buffer would be a genetic network employing split transcription factors, ${ }^{36}$ while this would simultaneously insulate the riboswitch. Here, the input-sensing riboswitch controls the translation of a hybrid activation domain $(\mathrm{AD})$ from a transcription factor such as GAL4. At the same time, a separate, cognate GAL4 DNAbinding domain (DBD) is transcribed at equal levels to the $\mathrm{AD}$ transcript (Figure 2). Thus, the DBD will be present in high numbers relative to the low number of DNA-binding sites positioned upstream of the output gene. The DBD thereby functions both as a mediator for the signal-correlated $\mathrm{AD}$ when bound to DNA, and as a surplus buffer molecule when DBD is not bound to DNA. Thus, the output would only be driven by the fraction of $\mathrm{DBD}_{\mathrm{DNA}}$ bound to an $\mathrm{AD}$ molecule. Due to the buffering capacity of surplus $\mathrm{DBD}$, this network architecture may also buffer against a few $\mathrm{AD}$ proteins unintentionally translated due to leakage or possible intermittent absence of riboswitch input in the riboswitch signals.

Mathematical Model of a Synthetic Signal Buffer System. We constructed a simple mathematical model for the conceived buffer network to guide the design. The model was built through application of ordinary differential equations (ODEs) describing the accumulation of mRNA and protein for the system (Table 1).

On the basis of equilibrium reactions between receptor $\mathrm{R}$ and ligand L, we derived simple Michaelis-Menten type saturation fractions $(f)$ for receptor saturations given the formal $\mathrm{L}$ and $\mathrm{R}$ concentrations and their dissociation constant $\left(K_{\mathrm{d}}\right)$ (more details in Supporting Information). Receptors and ligands notably represent several interactions in the network: riboswitch:tetracycline, DNA:DBD, DBD:AD.

The saturation fractions of these regulating receptors were subsequently used in the ODEs to linearly regulate the formation rates $\left(V_{\text {form }}\right)$ of cognate mRNA and protein (i.e., the transcription and translation rates). Formation of a regulated species was further limited by a minimum and maximum value, describing a leakiness level, and a general 

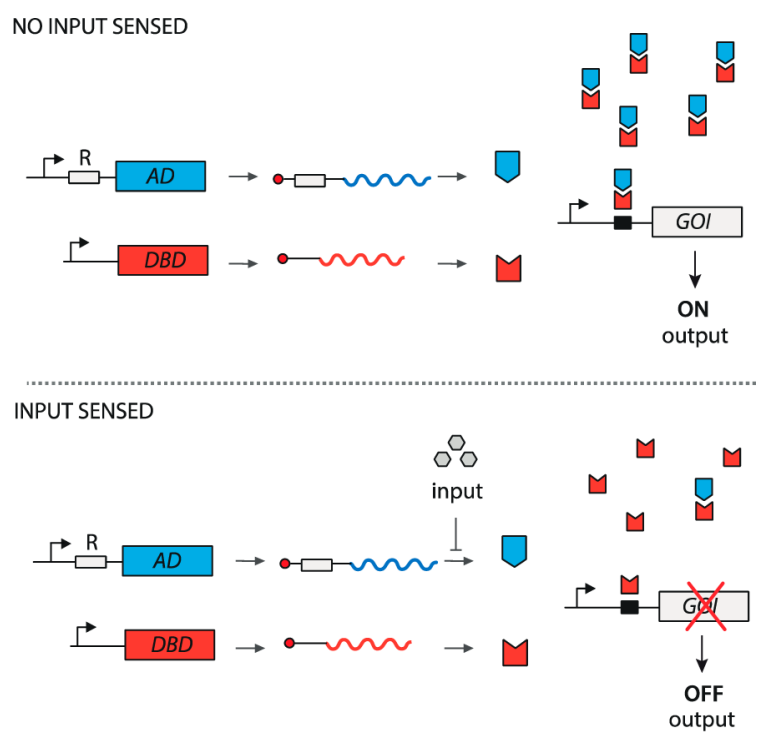

$\stackrel{R}{\square}$ Riboswitch $\quad$ DBD binding sites

Figure 2. Design of the molecular buffer network to modulate riboswitch signals. The signal-buffer design is composed of equal-level expression of the two independent GAL4 transcription factor domains, the riboswitch-regulated $\mathrm{AD}$ (blue) and the $\mathrm{DBD}$ (red), both fused to mutually interacting domains. The output gene of interest (GOI) is expressed from a minimal promoter featuring upstream binding sites for the DBD. When adding riboswitch ligand as input to the system, the translation of $\mathrm{AD}$ mRNA is inhibited, leading to a reduced expression of the GOI. The buffer system thus relays the riboswitch signal, while also insulating the output GOI.

reduction in translation strength, e.g., as observed for riboswitches. $^{37}$
In the case of the buffer system, the general expression for $f$ can both be used to model the fraction of DNA sites that is bound to $\mathrm{DBD}$ and the fraction of DBD that is bound to $\mathrm{AD}$, assuming simple binding dynamics. Since we assume that the two interactions are independent of each other, we can multiply the two fractions to report the fraction of DNA sites that is bound by an $\mathrm{AD}-\mathrm{DBD}$ complex, or the fraction of time that a given $\mathrm{DNA}$ site will be bound by an $\mathrm{AD}-\mathrm{DBD}$ complex.

According to the model, the specific tuning of the buffer molecule levels significantly impacts function, and full utilization of the riboswitch regulation potential will be achieved with roughly equal expression of $\mathrm{DBD}$ and $\mathrm{AD}$ transcripts (Figure S3).

Molecular Construction of the Synthetic Signal Buffer System. To set up the buffer network in S. cerevisiae, cognate GAL4 $\mathrm{AD}$ and $\mathrm{DBD}$ proteins were expressed with equal strength from $A D H 1$ promoters with the tetracyclineresponsive riboswitch down-regulating translation of $\mathrm{AD}$ (Figure 2). Repeats of the cognate DNA-binding sites were positioned upstream of a minimal promoter from SPO13. This promoter features a natural UME6 repressor binding site to reduce system-independent expression, such as previously engineered for a yeast two-hybrid strain (SPAL10 in strain $\mathrm{MaV203})^{38}$ to allow GAL4-dependent regulation of URA3 in a range affecting colony formation. After relaying the riboswitch signal through the buffer network, population-level control of the URA3 phenotypes became possible in an S. cerevisiae strain deficient of wild type GAL4 and GAL80 (Figure 3A). The signal-buffered strains acquired an ability to link colony formation to presence of the riboswitch input using classical $\mathrm{FOA}^{\mathrm{R}}$ selection where false-positive cells were first observed when spotting $10^{5}$ cells (Figure $3 \mathrm{~A}$ ) in the buffered strain. Further, since all plated cells formed colonies in the presence of

Table 1. Ordinary Differential Equations Describing the Formation and Degradation of mRNA and Protein in the Split TF Buffer System, Given the Saturation Fractions $f$ of Regulating Receptors (Riboswitch or TF)

\begin{tabular}{|c|c|}
\hline species & differential equation \\
\hline $\mathrm{AD}$ mRNA & $\frac{\mathrm{d}[\mathrm{AD} \mathrm{mRNA}]}{\mathrm{d} t}=V_{\text {form }}-k_{\mathrm{degr}_{\mathrm{RNA}}} \cdot[\mathrm{AD} \mathrm{mRNA}]$ \\
\hline DBD mRNA & $\frac{\mathrm{d}[\mathrm{DBD} m \mathrm{mNA}]}{\mathrm{d} t}=V_{\mathrm{form}}-k_{\mathrm{degr}_{\mathrm{RNA}}} \cdot[\mathrm{DBD} \mathrm{mRNA}]$ \\
\hline URA3 mRNA (driven by DBD:AD) & $\begin{aligned} \frac{\mathrm{d}[\mathrm{URA} 3 \mathrm{mRNA}]}{\mathrm{d} t}= & \mathrm{GAL}_{\min }+\left(\mathrm{GAL}_{\max }-\mathrm{GAL}_{\min }\right) \cdot f_{A D D B D} \cdot f_{D B D D N A} \\
& -k_{\operatorname{degr}_{\mathrm{RNA}}} \cdot[\mathrm{URA} 3 \mathrm{mRNA}]\end{aligned}$ \\
\hline $\mathrm{AD}$ protein & $\frac{\mathrm{d}[\mathrm{AD}]}{\mathrm{d} t}=\left(\mathrm{ribo}_{\max }-\left(\mathrm{ribo}_{\max }-\mathrm{ribo}_{\min }\right) \cdot f_{\text {ribo }}\right) \cdot[\mathrm{AD} \mathrm{mRNA}]-k_{\mathrm{deg}_{\text {prot }}}[\mathrm{AD}]$ \\
\hline DBD protein & $\frac{\mathrm{d}[\mathrm{DBD}]}{\mathrm{d} t}=k_{\text {form }} \cdot[\mathrm{DBD} \mathrm{mRNA}]-k_{\mathrm{deg}_{\mathrm{prot}}}[\mathrm{DBD}]$ \\
\hline GAL4 mRNA & $\frac{\mathrm{d}[\mathrm{GAL} 4 \mathrm{mRNA}]}{\mathrm{d} t}=V_{\text {form }}-k_{\text {degr }_{\mathrm{RNA}}} \cdot[\mathrm{GAL} 4 \mathrm{mRNA}]$ \\
\hline GAL4 protein & $\begin{aligned} \frac{\mathrm{d}[\mathrm{GAL} 4]}{\mathrm{d} t}= & \left(\mathrm{ribo}_{\max }-\left(\mathrm{ribo}_{\max }-\mathrm{ribo}_{\min }\right) \cdot f_{\text {ribo }}\right) \cdot[\mathrm{GAL} 4 \mathrm{mRNA}] \\
& -k_{\mathrm{degr}_{\text {prot }}}[\mathrm{GAL} 4]\end{aligned}$ \\
\hline URA3 mRNA (driven by GAL4) & $\begin{aligned} \frac{\mathrm{d}[\mathrm{URA} 3 \mathrm{mRNA}]}{\mathrm{d} t}= & \mathrm{GAL}_{\min }+\left(\mathrm{GAL}_{\max }-\mathrm{GAL}_{\min }\right) \cdot f_{\mathrm{GAL}_{4 \mathrm{DNA}}} \\
& -k_{\mathrm{degr}_{\mathrm{RNA}}} \cdot[\mathrm{URA} 3 \mathrm{mRNA}]\end{aligned}$ \\
\hline URA3 protein & $\frac{\mathrm{d}[\mathrm{URA} 3]}{\mathrm{d} t}=k_{\text {form }} \cdot[\mathrm{URA} \mathrm{mRNA}]-k_{\mathrm{degr}_{\mathrm{prot}}} \cdot[\mathrm{URA} 3 \mathrm{mRNA}]$ \\
\hline
\end{tabular}




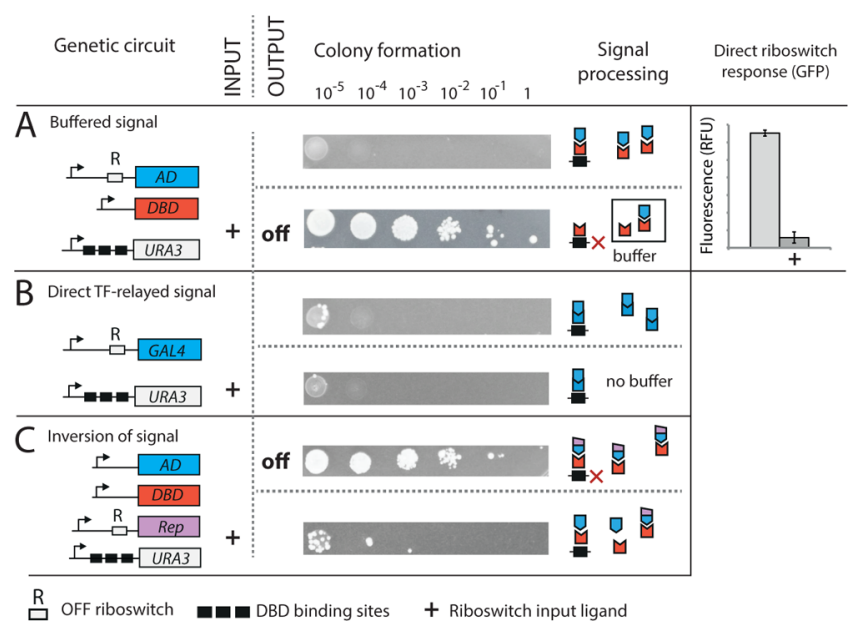

Figure 3. Colony formation in response to riboswitch-sensed inputs using the engineered circuits. (A) Signal-buffered riboswitch relay provided full ligand-controlled colony formation (PRa22). (B) Relay of the riboswitch through the single-protein wild type transcription factor did not allow ligand-dependent colony formation (PRa28). (C) Modular inversion of the riboswitch signal by regulation via the GAL80 repressor (PRa84). The riboswitch (R) was expressed in the 5 -untranslated region of the illustrated target gene $(\mathrm{AD}, \mathrm{GAL} 4$ or the GAL80 repressor). Colony-formation responses were determined using spot assays with 10-fold serial dilution of the cultures (Methods) and representative examples of triplicate tests shown. GFP output was measured as background-subtracted relative fluorescence units (RFU) measured from a C-terminally GFP-tagged $\mathrm{AD}$ (PRa115), with error bars depicting standard error $(n=3)$. Plates were SC-leu-trp, with $0.09 \%(\mathrm{w} / \mathrm{v})$ FOA in selective medium. $150 \mu \mathrm{M}$ tetracycline was used as input ligand in plates, $150 \mu \mathrm{M}$ tetracycline in liquid cultures.

tetracycline (Figure 3A), both false-positive and false-negative colonies were reduced at the same time using the molecular buffer compared to the strain directly linking the signal from the riboswitch sensor to the output gene (Figure 1). We also observed efficient tetracycline-dependent down-regulation of $\mathrm{AD}$ by $\mathrm{C}$-terminal GFP tagging, confirming that the riboswitch signal was relayed when using the $\mathrm{AD}$ gene (Figure $3 \mathrm{~A}$ ). Thus, insulated from the URA3 gene, the riboswitch signal propagated correctly.

We hypothesized that the introduction of a buffering layer can provide additional, modular advantages for signal tuning unlike a possible insulation using a simple $\mathrm{N}$-terminal tag. However, the complete network also must be carefully balanced and thus we first tested the importance of the introduced DBD buffer molecules compared to simply passing the signal through a transcription factor layer employed at the same transcriptional strength but without buffering. Likely due to the high expression level and concomitant constitutive DNA saturation, use of a single-protein, wild type GAL4 transcription factor resulted in no control of colony formation (Figure 3B) despite possessing the same DNA-binding domain (as modeled Figure S4). As indicated in a riboswitch selection study, such direct riboswitch control of a full transcription factor likely requires use of a much weaker expression level (driven by CYC1 promoter). ${ }^{39}$

Modular Inversion of Sensor Signals. The need for ON or OFF switches in genetic circuits depends on the desired actuations and outputs. To allow the combination of OFF riboswitches with gain-of-function genes, we wanted to demonstrate the ease by which riboswitch signals can be treated modularly with the synthetic buffer network and a repressor module. Engineering of regulated repression by adding repressor-binding sites within synthetic/hybrid promoters can be challenging. Instead we took advantage of the constructed, robust activation modules and inverted the signal modularly by relaying the OFF signal to the GAL80 antiactivating repressor, which binds and inhibits GAL4 AD. ${ }^{40}$ In this way signal inversion results from expressing the genes encoding $\mathrm{DBD}$ and $\mathrm{AD}$ with the same transcriptional strength as a gene encoding GAL80 controlled by the tetracycline
A

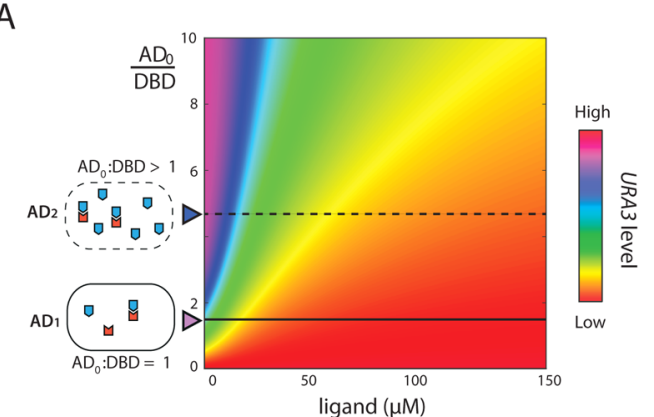

C

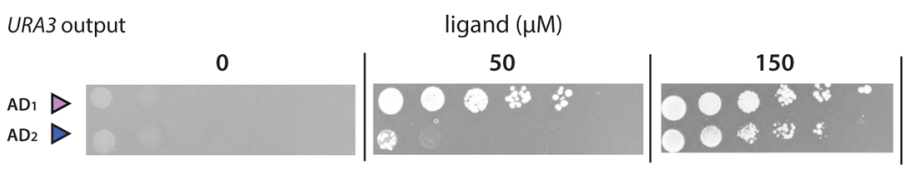

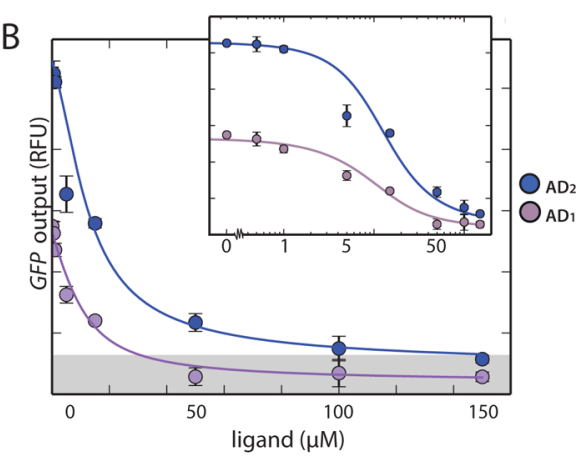

Figure 4. Tuning the effective sensitivity for the ligand by modulating the transcriptional ratio of $\mathrm{AD}$ and DBD in the buffered network with fluorescence and colony formation as output. (A) Model showing the effect on output (URA3 expression) by changing the AD:DBD transcriptional ratio $\left(\mathrm{AD}_{0}: \mathrm{DBD}\right)$ or adding riboswitch ligand. (B) Dynamic range tuning measured in S. cerevisiae with 6xGFP as output. Background-subtracted relative fluorescence units (RFU) are shown responding to added riboswitch ligand (tetracycline) for two strains $A D_{1}$ and $A D_{2}$ with perturbed $\mathrm{AD}_{0}: \mathrm{DBD}$, error bars denote std. error $(n=3)$. The output is shown with the fit to the model with linear $x$-axis and in the small window with logarithmic $x$-axis. (C) Shifted trigger point for colony formation of the two perturbed strains spotted in 10-fold serial-dilution spot assays of equally dense cultures on SC-leu-trp $+0.09 \%$ FOA with indicated riboswitch ligand concentrations (tetracycline) (one representative experiment shown from triplicates). 
riboswitch. With these strains using the exact same sensor and output modules, the opposite colony formation behavior was observed (Figure 3C) compared to the first buffered strain, although the frequency of false positives increased by 10 -fold, indicating that the expression level of GAL80 could be further optimized relative to $\mathrm{DBD}$ and $\mathrm{AD}$.

Using the Synthetic Buffer Network for Modular Tuning of Input Sensitivity. The sensitivity of a riboswitch for its ligand depends on molecular RNA interactions, which are nontrivial to engineer. ${ }^{25}$ Instead, our model suggested that the sensitivity could be modularly shifted by perturbing the transcriptional ratios of $\mathrm{AD}$ to $\mathrm{DBD}\left(\mathrm{AD}_{0}: \mathrm{DBD}\right)$ while maintaining the riboswitch unchanged (Figure 4A). We calculated steady state concentrations of URA3 at different tetracycline concentrations and transcriptional $\mathrm{AD}_{0}: \mathrm{DBD}$ ratios by setting the ODEs equal to zero. The $\mathrm{AD}_{0}: \mathrm{DBD}$ ratio was varied by changing the $\mathrm{DBD}$ transcription rate while keeping $\mathrm{AD}$ transcription rate constant.

By introducing more free $\mathrm{AD}$ molecules relative to $\mathrm{DBD}$ in the system, a higher number of input molecules (i.e., larger $\mathrm{AD}$ reduction) would be required to lead to the same output. Thus, an increased ligand concentration would be required in order to produce an $\mathrm{AD}: \mathrm{DBD}$ protein ratio that outputs a survival response (Figure $4 \mathrm{~A}$ ).

To test this experimentally, we perturbed the ratios by introducing the weaker CUP1 promoter to drive DBD expression as a low-expression alternative to the $A D H 1$ promoter $^{41}$ otherwise used. The response curves of the two perturbed systems measured with $2 \times 3$ tandem green fluorescent protein $(2 \times 3 \mathrm{vGFP})^{42}$ displayed this response shift and the changed curvature was fitted to our model (Figure 4B) with high confidence $\left(R^{2}=0.98\right.$ and 0.96$)$. The increase of $\mathrm{AD}: \mathrm{DBD}$ transcriptional ratio resulted in a vertical response increase, probably due to a higher degree of $\mathrm{AD}$-binding to DNA-bound DBD. More interestingly and as predicted, it also introduced a horizontal right-shift, thus increasing the number of ligand molecules needed to produce the same absolute expression level and produce the same relative down-regulation from the maximum. This horizontal shift could also be relayed to colony-formation output (Figure 4C). The increased $\mathrm{AD}: \mathrm{DBD}$ transcription ratio effectively shifted the ligand sensitivity threshold to trigger survival at an increased ligand concentration. Whereas the strain $\mathrm{AD}_{1}$ with $A D H 1$-based expression of $\mathrm{AD}$ and $\mathrm{DBD}$ required $50 \mu \mathrm{M}$ tetracycline to trigger the survival, the strain $\mathrm{AD}_{2}$ with increased $\mathrm{AD}$ :DBD transcription ratio required $150 \mu \mathrm{M}$ tetracycline to trigger survival (Figure 4C). As predicted by the model, the low threshold concentration could be reconstituted by simply reducing the absolute transcription levels for $\mathrm{AD}$ as much as for $\mathrm{DBD}$, hence reestablishing the 1:1 transcriptional $\mathrm{AD}: \mathrm{DBD}$ ratio (Figure S5). Much like $\mathrm{pH}$ buffers, the output did not change notably in response to absolute changes in the concentration of the interacting buffer pairs when their mutual ratio was kept the same.

\section{DISCUSSION}

In next-generation synthetic systems, biological signal interfaces that improve parts' interoperability are needed to meet the challenge of designing diverse biological functions using diverse biological parts. Recently, such progress has been attained using spatial insulators to limit the negative impact from the genetic context of the combined parts ${ }^{35}$ or using modular signal transduction scaffolds with autoinhibition. ${ }^{43}$ In other systems, better $\mathrm{I} / \mathrm{O}$ coherence has been obtained through use of directed evolution approaches or extensive tuning libraries sampling the functional circuit space. ${ }^{15,44}$ Another powerful method for signal improvement is the use of protein sequestration to generate ultrasensitivity and to transform graded signals into binary forms. ${ }^{30,45}$ Ultrasensitivity can result if the buffering molecules have higher affinity for the input signal than the output relay has, ${ }^{46}$ whereas in our demonstration, the buffering agent is the same protein as the output relay. Molecular buffering may be a natural signal stabilization strategy. In fact, buffering of noise in some natural systems has been predicted as a result of the order of dimer transcription factor binding, which produces a pool of signal stabilizing, inactive monomers. ${ }^{47}$ In this study, we demonstrated that design principles of molecular buffers can be reconstructed synthetically to effectively tune and utilize the signal response of a riboswitch, allowing new cellular actuations, while also insulating the riboswitch from the nucleotide context. Adapted from $\mathrm{pH}$-stabilizing buffers, this proteinbased buffer device allowed the modular tuning of riboswitch signals. Similarly, the input trigger threshold for shifting the output phenotype could be tuned by changing the ratio of the buffer proteins, analogous to how $\mathrm{pH}$ buffer ratios affect the stabilized $\mathrm{pH}$.

Signal modulation has been described employing different pools of "unfunctional" response mediators such as antiactivators and shunt DNA-binding sites to change GFP-based outputs. $^{30,48,49}$ Introducing signal computation based on protein-protein interactions, this concept alleviates issues with tuning the expression level of DNA-binding proteins such as repressors at levels of a few molecules per cell where unintended, constitutive oversaturation of the binding sites will result in loss of signal. Using split transcription factors, we instead rely on customized protein-protein interaction of hybrid proteins. Lower binding affinity between these parts allows responses to transmit at higher signal molecule concentrations, which may provide stability toward fluctuations. These provide an easy protein-protein interaction control point for inversion of the signal direction, which is often important as many output genes work only with ON signals.

These different generic interaction interfaces of the network may serve to further incorporate multicomponent signal schemes comprising, e.g., sub-buffer systems by engineering specific conditional DBDs and specific protein-interaction domains without module cross talk. We also anticipate that the signal-stabilizing network engineered in this study could be reconstructed in quite different synthetic embodiments by implementation of other buffer molecules cognate to an otherwise fluctuating signal. By taking advantage of the natural concept of molecular signal buffers, these systems will aid the large-scale domestication of wild type or synthetic input sensors for more predictable, customized cell reprogramming.

\section{METHODS}

Standard methods for strain construction and molecular biology in S. cerevisiae and Escherichia coli were used. All plasmids and chromosomal deletion substrates cloned in this study were constructed using uracil-excision cloning ${ }^{50}$ by assembly of PCR fragments as described in Supporting Information. Complete cloning and strain construction methods, strain lists and plasmid lists are given in Supporting Information. 
Colony Formation Response Assays. Four mL synthetic complete (SC) medium ( $2 \%$ glucose, $\mathrm{pH}=5.6$ ) lacking leucine (leu) and tryptophan (trp) was inoculated with a single colony of the strain and split into two halves for preculturing with/ without the riboswitch ligand ( $150 \mu \mathrm{M}$ tetracycline) for $18 \mathrm{~h}$ at $30{ }^{\circ} \mathrm{C}, 175 \mathrm{rpm}$ horizontal shaking. Each culture was 10-fold serial diluted in a 96-well plate, such that each dilution contained $100 \mu \mathrm{L}$ of volume. Five $\mu \mathrm{L}$ of each dilution (both precultures) was spotted onto SC-leu-trp plates $(\mathrm{pH}=4.5)$ and the respective assay plates, supplemented with $0.09 \%(\mathrm{w} /$ v) FOA and the relevant concentration of tetracycline. Preparation of FOA-containing plates is further described in Supporting Information. Plates were incubated in darkness at $30{ }^{\circ} \mathrm{C}$ for 3 days. For spot assays, equal cell concentrations between strains and conditions were controlled by evaluation of the spots on SC -leu -trp plates. Photographs were taken with a ColonyDoc-It (UVP).

GFP Response Assays. Precultures of the strains PRa74 (background), PRa78 $\left(\mathrm{AD}_{1}\right)$ and PRa79 $\left(\mathrm{AD}_{2}\right)$ were inoculated from a single colony of the strain in SC medium ( $2 \%$ glucose, $\mathrm{pH}=5.6$ ) lacking leu, trp and histidine. Following $18 \mathrm{~h}$ of cultivation at $30{ }^{\circ} \mathrm{C}, 175 \mathrm{rpm}$ horizontal shaking, $200 \mu \mathrm{L}$ microtiter main cultures were inoculated from these in $75 \%$ SC medium (diluted with Milli-Q water and back-standardized to $2 \%$ glucose) with the relevant concentrations of tetracycline added. The cultures were sealed with a gas-permeable Breathseal (Greiner bio-one) and plastic lid and were cultured in a horizontal shaker (Innova) at $30{ }^{\circ} \mathrm{C}, 300 \mathrm{rpm}$ shaking. Following $16 \mathrm{~h}$ of cultivation, the cultures were measured by flow cytometry on a BD LSRFortessa Cell Analyzer using a FITC filter with collection limit set to 10000 cells. The mean FITC intensity for each sample was reported. The measurements from the GFP-devoid PRa74 strain were used for background-subtraction.

Direct Riboswitch Response (GFP-Tagging). Precultures of the strains PRa18 (background), PRa115 (AD-GFP) and PRa116 (URA3-GFP) were inoculated from a single colony of the strain in SC medium ( $2 \%$ glucose, $\mathrm{pH}=5.6)$ lacking the relevant auxotrophic selection dropout (leu or trp). Following $18 \mathrm{~h}$ of cultivation at $30{ }^{\circ} \mathrm{C}, 250 \mathrm{rpm}$ horizontal shaking, $4 \mathrm{~mL}$ cultures of SC medium ( $2 \%$ glucose, $\mathrm{pH}=5.6,250 \mu \mathrm{M} \mathrm{Cu}^{2+}$ ) were inoculated from the precultures (by $100 \times$ back-dilution) and with the relevant concentrations of tetracycline added. Following $16 \mathrm{~h}$ of cultivation at $30^{\circ} \mathrm{C}, 250 \mathrm{rpm}$ horizontal shaking, the cultures were measured in a Synergy H1 reader (BioTek) with $485 \mathrm{~nm}$ excitation, $528 \mathrm{~nm}$ emission and standardized to the cell density (OD600). The measurements from the GFP-devoid PRa18 strain were used for backgroundsubtraction.

\section{ASSOCIATED CONTENT}

\section{(S) Supporting Information}

The Supporting Information is available free of charge on the ACS Publications website at DOI: 10.1021/acssynbio.5b00213.

Detailed description of mathematical model; No regulation of output when controlling expression of GAL4; Re-establishing AD:DBD transcription ratio reestablishes response; Detailed methods and materials; Model (Python) script. (PDF)

\section{AUTHOR INFORMATION}

\section{Corresponding Author}

*E-mail: msom@bio.dtu.dk.

\section{Author Contributions}

P.R., H.J.G. and M.O.A.S. designed research; P.R. and KSL performed research; K.J. developed the mathematical model. All authors analyzed the results and wrote the manuscript.

\section{Notes}

The authors declare the following competing financial interest(s): P.R., H.J.G. and M.O.A.S are inventors on patent applications filed on the signal buffer system (EP2013 0186673 and WO2015 044456A1).

\section{ACKNOWLEDGMENTS}

We kindly thank Marius Boulos Faza and Luisa Gronenberg for helpful comments and Niels Bjerg Jensen for helpful suggestions on gene deletion. This research was funded by the Novo Nordisk Foundation and the European Union Seventh Framework Programme (FP7-KBBE-2013-7-singlestage) under Grant agreement no. 613745, Promys.

\section{REFERENCES}

(1) Jensen, M. K., and Keasling, J. D. (2014) Recent applications of synthetic biology tools for yeast metabolic engineering. FEMS Yeast Res., DOI: 10.1111/1567-1364.12185.

(2) Khalil, A. S., and Collins, J. J. (2010) Synthetic biology: applications come of age. Nat. Rev. Genet. 11, 367-79.

(3) Wang, Y.-H., Wei, K. Y., and Smolke, C. D. (2013) Synthetic Biology: Advancing the Design of Diverse Genetic Systems. Annu. Rev. Chem. Biomol. Eng. 4, 69-102.

(4) Wang, J., and Katz, E. (2010) Digital biosensors with built-in logic for biomedical applications-biosensors based on a biocomputing concept. Anal. Bioanal. Chem. 398, 1591-603.

(5) Michener, J. K., and Smolke, C. D. (2012) High-throughput enzyme evolution in Saccharomyces cerevisiae using a synthetic RNA switch. Metab. Eng. 14, 306-316.

(6) Weber, W., and Fussenegger, M. (2006) Pharmacologic transgene control systems for gene therapy. J. Gene Med. 8, 535-56.

(7) Sprinzak, D., and Elowitz, M. B. (2005) Reconstruction of genetic circuits. Nature 438, 443-8.

(8) Ausländer, S., and Fussenegger, M. (2013) From gene switches to mammalian designer cells: present and future prospects. Trends Biotechnol. 31, 155-68.

(9) Benenson, Y. (2012) Biomolecular computing systems: principles, progress and potential. Nat. Rev. Genet. 13, 455-68.

(10) Galvão, T. C., and Lorenzo, V. de. (2006) Transcriptional regulators à la carte: engineering new effector specificities in bacterial regulatory proteins. Curr. Opin. Biotechnol. 17, 34-42.

(11) Eggeling, L., Bott, M., and Marienhagen, J. (2015) Novel screening methods-biosensors. Curr. Opin. Biotechnol. 35, 30-36.

(12) Mandal, M., and Breaker, R. R. (2004) Gene regulation by riboswitches. Nat. Rev. Mol. Cell Biol. 5, 451-63.

(13) Berens, C., Groher, F., and Suess, B. (2015) RNA aptamers as genetic control devices: The potential of riboswitches as synthetic elements for regulating gene expression. Biotechnol. J. 10, 246-57.

(14) Xie, Z., Wroblewska, L., and Prochazka, L. (2011) Multi-input RNAi-based logic circuit for identification of specific cancer cells. Science (Washington, DC, U. S.) 333, 1307-1311.

(15) Yokobayashi, Y., Weiss, R., and Arnold, F. H. (2002) Directed evolution of a genetic circuit. Proc. Natl. Acad. Sci. U. S. A. 99, 1658791.

(16) Cantone, I., Marucci, L., Iorio, F., Ricci, M. A., Belcastro, V., Bansal, M., Santini, S., di Bernardo, M., di Bernardo, D., and Cosma, M. P. (2009) A yeast synthetic network for in vivo assessment of reverse-engineering and modeling approaches. Cell 137, 172-81. 
(17) Kaern, M., Blake, W. J., and Collins, J. J. (2003) The engineering of gene regulatory networks. Annu. Rev. Biomed. Eng. 5, 179-206.

(18) Stanton, B. C., Nielsen, A. a K., Tamsir, A., Clancy, K., Peterson, T., and Voigt, C. a. (2014) Genomic mining of prokaryotic repressors for orthogonal logic gates. Nat. Chem. Biol. 10, 99-105.

(19) Stoltenburg, R., Reinemann, C., and Strehlitz, B. (2007) SELEX-a (r)evolutionary method to generate high-affinity nucleic acid ligands. Biomol. Eng. 24, 381-403.

(20) Win, M. N., and Smolke, C. D. (2008) Higher-order cellular information processing with synthetic RNA devices. Science 322, 45660 .

(21) Rodrigo, G., Landrain, T. E., and Jaramillo, A. (2012) De novo automated design of small RNA circuits for engineering synthetic riboregulation in living cells. Proc. Natl. Acad. Sci. U. S. A. 109, 152716.

(22) Lucks, J. B., Qi, L., Mutalik, V. K., Wang, D., and Arkin, A. P. (2011) Versatile RNA-sensing transcriptional regulators for engineering genetic networks. Proc. Natl. Acad. Sci. U. S. A. 108, 8617-22.

(23) Davidson, E. A., and Ellington, A. D. (2007) Synthetic RNA circuits. Nat. Chem. Biol. 3, 23-8.

(24) Isaacs, F. J., Dwyer, D. J., and Collins, J. J. (2006) RNA synthetic biology. Nat. Biotechnol. 24, 545-54.

(25) Soulière, M., Altman, R., Schwarz, V., Haller, A., Blanchard, S., and Micura, R. (2013) Tuning a riboswitch response through structural extension of a pseudoknot. Proc. Natl. Acad. Sci. U. S. A. 110, E3256-E3264.

(26) Green, A. A., Silver, P. A., Collins, J. J., and Yin, P. (2014) Resource Toehold Switches: De-Novo-Designed Regulators of Gene Expression. Cell 159, 925-939.

(27) Chappell, J., Takahashi, M. K., and Lucks, J. B. (2015) Creating small transcription activating RNAs. Nat. Chem. Biol. 11, 214-220.

(28) Muranaka, N., Abe, K., and Yokobayashi, Y. (2009) Mechanismguided library design and dual genetic selection of synthetic OFF riboswitches. ChemBioChem 10, 2375-81.

(29) Berens, C., and Suess, B. (2015) Riboswitch engineering making the all-important second and third steps. Curr. Opin. Biotechnol. 31, 10-15.

(30) Buchler, N. E., and Cross, F. R. (2009) Protein sequestration generates a flexible ultrasensitive response in a genetic network. Mol. Syst. Biol. 5, 272.

(31) Ang, J., Harris, E., and Hussey, B. (2013) Tuning Response Curves for Synthetic Biology. ACS Synth. Biol. 2, 547-567.

(32) Bellí, G., Garí, E., Piedrafita, L., Aldea, M., and Herrero, E. (1998) An activator/repressor dual system allows tight tetracyclineregulated gene expression in budding yeast. Nucleic Acids Res. 26, 942947.

(33) Zalatan, J. G., Lee, M. E., Almeida, R., Gilbert, L. A., Whitehead, E. H., La Russa, M., Tsai, J. C., Weissman, J. S., Dueber, J. E., Qi, L. S., and Lim, W. A. (2014) Engineering Complex Synthetic Transcriptional Programs with CRISPR RNA Scaffolds. Cell 160, 339-350.

(34) Kötter, P., Weigand, J. E., Meyer, B., Entian, K.-D., and Suess, B. (2009) A fast and efficient translational control system for conditional expression of yeast genes. Nucleic Acids Res. 37, e120.

(35) Lou, C., Stanton, B., Chen, Y.-J., Munsky, B., and Voigt, C. a. (2012) Ribozyme-based insulator parts buffer synthetic circuits from genetic context. Nat. Biotechnol. 30, 1137.

(36) Rugbjerg, P., Myling-Petersen, N., and Sommer, M. O. A. (2015) Flexible metabolic pathway construction using modular and divisible selection gene regulators. Metab. Eng. 31, 189-197.

(37) Weigand, J. E., Wittmann, A., and Suess, B. (2012) Synthetic Gene Networks. Methods Mol. Biol. 813, 157-168.

(38) Vidal, M., Brachmann, R. K., Fattaey, a, Harlow, E., and Boeke, J. D. (1996) Reverse two-hybrid and one-hybrid systems to detect dissociation of protein-protein and DNA-protein interactions. Proc. Natl. Acad. Sci. U. S. A. 93, 10315-20.

(39) Klauser, B., Atanasov, J., Siewert, L. K., and Hartig, J. S. (2015) Ribozyme-Based Aminoglycoside Switches of Gene Expression Engineered by Genetic Selection in S. cerevisiae. ACS Synth. Biol. 4, 516.
(40) Traven, A., Jelicic, B., and Sopta, M. (2006) Yeast Gal4: a transcriptional paradigm revisited. EMBO Rep. 7, 496-9.

(41) Barbour, L., Zhu, Y., and Xiao, W. (2000) Improving synthetic lethal screens by regulating the yeast centromere sequence. Genome 43, 910-17.

(42) Rugbjerg, P., Knuf, C., Förster, J., and Sommer, M. O. a. (2015) Recombination-stable multimeric green fluorescent protein for characterization of weak promoter outputs in Saccharomyces cerevisiae. FEMS Yeast Res. 15, fov085.

(43) Whitaker, W. R., Davis, S. A., Arkin, A. P., and Dueber, J. E. (2012) Engineering robust control of two-component system phosphotransfer using modular scaffolds. Proc. Natl. Acad. Sci. U. S. A. 109, 18090-18095.

(44) Egbert, R. G., and Klavins, E. (2012) Fine-tuning gene networks using simple sequence repeats. Proc. Natl. Acad. Sci. U. S. A. 109, $16817-22$.

(45) Palani, S., and Sarkar, C. A. (2011) Synthetic conversion of a graded receptor signal into a tunable, reversible switch. Mol. Syst. Biol. 7,480 .

(46) Buchler, N. E., and Louis, M. (2008) Molecular titration and ultrasensitivity in regulatory networks. J. Mol. Biol. 384, 1106-19.

(47) Bundschuh, R., Hayot, F., and Jayaprakash, C. (2003) The Role of Dimerization in Noise Reduction of Simple Genetic Networks. J. Theor. Biol. 220, 261-269.

(48) Daniel, R., Rubens, J. R., Sarpeshkar, R., and Lu, T. K. (2013) Synthetic analog computation in living cells. Nature 497, 619-23.

(49) Chen, D., and Arkin, A. P. (2012) Sequestration-based bistability enables tuning of the switching boundaries and design of a latch. Mol. Syst. Biol. 8, 620.

(50) Nour-Eldin, H. H., Hansen, B. G., Nørholm, M. H. H., Jensen, J. K., and Halkier, B. a. (2006) Advancing uracil-excision based cloning towards an ideal technique for cloning PCR fragments. Nucleic Acids Res. 34, e122. 